\section{Lower Gastrointestinal Bleeding from Aneurysmo-Colonic Fistula after Endovascular Aneurysm Repair}

\section{Introduction}

We report a rare case of internal iliac artery aneurysmo-colonic fistula that developed after endovascular aneurysm repair (EVAR) and transarterial embolization (TAE) in the distal internal iliac artery. An 81-year-old man with a history of internal iliac artery aneurysm (IIAA) received TAE and EVAR. He developed hydronephrosis 7 months after the operation, so he received a percutaneous nephrostomy (PCN). Thereafter, he developed hematuria persisting for more than 2 years. Moreover, hematochezia occurred 2 years after the hematuria episode. Owing to the internal iliac artery aneurysmocolonic fistula, hematochezia developed subsequently.

\section{Case Report}

An 81-year-old man presented to the emergency department with a 2-week history of repeated bloody stools. In March 2015, he had right internal iliac artery aneurysm rupture (Panel A, arrow). $\mathrm{He}$ received EVAR and TAE in the distal internal iliac artery. In the next month, he had a retroperitoneal abscess, which was confirmed by the presence of Escherichia coli in blood culture and computed tomography (Panel B, red arrow). In addition, computed tomography revealed right hydronephrosis. After he accepted percutaneous abscess drainage, he recovered well. However, he found that he had hematuria in October 2015. He underwent percutaneous nephrostomy to improve his symptom. Thereafter, he also underwent revision of the percutaneous nephrostomy tube monthly due to failure of the double-J stent setting. In September 2016, he developed sepsis due to E. coli infection. Moreover, he had repeated bloody stools since April 2017. He underwent a colonoscopic examination five times because we could not make a definitive diagnosis. Colonoscopy revealed a sigmoid colon ulcer and active bleeding. We could not finish the hemostatic procedure, so we suspected the formation of fistula between the aneurysm and the sigmoid colon. Consequently, computed tomography was arranged and revealed aneurysm involvement to the adjacent colon with possible fistula formation (Panel C, arrow). Therefore, he underwent operation with the Hartmann procedure. The pathological report demonstrated that the picture was compatible with aneurysmo-colonic fistula (Panel D, black arrow: aorta wall, green arrow: colon).

\section{Discussion}

As for IIAA, we can choose open repair or endovascular repair However, compared with open repair, EVAR is associated with fewer complications [1]. We mainly chose to perform EVAR for our patient according to his clinical condition. We also implanted a stent graft in the right internal iliac artery [2]. After the operation, we needed to combine antibiotic treatment with abscess drainage [3]. A

\section{Journal of}

Emergency Medicine \& Critical Care

\author{
Yuan-Jung Chen ${ }^{1 *}$, Chun-Han Shih ${ }^{2}$, Yuan-Yao \\ Tsai ${ }^{1}$ and Hui-Han Lin ${ }^{3}$ \\ ${ }^{\prime}$ Divisions of Gastroenterology and Colorectal Surgery, China Medical \\ University Hospital, China Medical University, Taiwan, China \\ ${ }^{2}$ Division of Gastroenterology, China Medical University Hospital, China \\ Medical University, Taiwan, China \\ ${ }^{3}$ Division of Cardiovascular Surgery, China Medical University Hospital, China \\ Medical University, Taiwan, China

\section{Address for Correspondence} \\ Yuan-Jung Chen, Divisions of Gastroenterology and Colorectal \\ Surgery, China Medical University Hospital, China Medical \\ University, Taiwan, China, E-mail: u102022028@cmu.edu.tw \\ Submission: 29 June, 2018 \\ Accepted: 30 July, 2018 \\ Published: 09 August, 2018 \\ Copyright: ๑ 2018 Chen YJ, et al. This is an open access article distributed \\ under the Creative Commons Attribution License, which permits unrestricted \\ use, distribution, and reproduction in any medium, provided the original work \\ is properly cited.
}

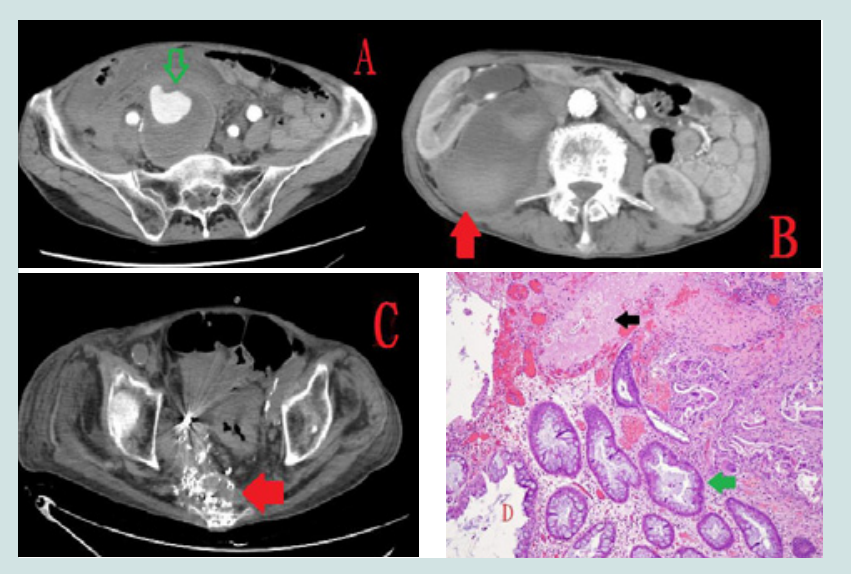

retroperitoneal abscess developed after the operation for EVAR. In fact, various forms of fistula could lead to hematuria [4-7]. Moreover, our patient found bloody stools 2 years later. The recurrent E. coli infection from the gastrointestinal tract devastatingly contributed to the internal iliac artery aneurysmo-colonic fistula. In most cases, we often find an aneurysmo-colonic fistula and aortoduodenal fistulas in some cases [8-14]. Antibiotic treatment should be combined with abscess drainage after the operation for EVAR. Otherwise, the abscess may create a fistula between ureter, bladder, and colon. A patient may show symptoms of hematuria or bloody stools.

\section{References}

1. Rana MA, Kalra M, Oderich GS, de Grandis E, Gloviczki P, et al. (2014) Outcomes of open and endovascular repair for ruptured and nonruptured internal iliac artery aneurysms. J Vasc Surg 59: 634-644.

2. Sedivy P, Spacek M, El Samman K, Belohlavek O, Mach T, et al. (2012) Endovascular treatment of infected aortic aneurysms. Eur J Vasc Endovasc 
Citation: Chen YJ, Shih CH, Tsai YY, Lin HH. Lower Gastrointestinal Bleeding from Aneurysmo-Colonic Fistula after Endovascular Aneurysm Repair. J Emerg Med Critical Care 2018;4(2): 2.

ISSN: $2469-4045$

Surg 44: 385-394.

3. Nakajima K, Kato N, Hashimoto T, Chino S, Higashigawa T, et al. (2018) Treatment of infected aneurysm with combined endovascular aneurysm repair and abscess drainage. J Vasc Interv Radiol 29: 188-193.

4. Métairie S, Denimal F, Floch I, Pillet JC, Pittaluga P, et al. (2001) Rupture of internal iliac artery aneurysm into the bladder following aortic aneurysm repair. Ann Vasc 15: 693-695.

5. Makatsori A, Stavropoulos NE (2010) Hematuria secondary to an internal iliac artery aneurysm. Korean J Urol 51: 583-585.

6. Cohen-Kashi KJ, Anne N, Pallapothu RK, Scher L (2006) Spontaneous rupture of hypogastric artery aneurysm into the bladder: a case report and review of the literature. Ann Vasc Surg 20: 134-137.

7. Honma I, Takagi Y, Shigyo M, Sunaoshi K, Inaoka M, et al. (2002) Massive hematuria after cystoscopy in a patient with an internal iliac artery aneurysm. Int J Urol 9: 407-409.

8. Khalaf C, Houlind KC (2017) Case report: primary aortosigmoid fistula - a rare cause of lower gastrointestinal bleeding. Int J Surg Case Rep 40: 20-22.
9. O'Brien J, Muscara F, Farghal A, Shaikh I (2016) Haematochezia from a splenic artery pseudoaneurysm communicating with transverse colon: a case report and literature review. Case Rep Vasc Med 2016: 1-5.

10. Policha A, Baldwin M, Mussa F, Rockman C (2015) lliac artery-uretero-colonic fistula presenting as severe gastrointestinal hemorrhage and hematuria: a case report and review of the literature. Ann Vasc Surg 29: 1656.

11. Katoh J, Shindo S, Kina S, Katahira S, Osawa H, et al. (1995) Rupture of an isolated internal iliac artery aneurysm into the rectum: report of a case. Surg Today 25: 554-556.

12. Karkos CD, Oshodi TO, Vimalachandran D, Abraham JS, Adiseshiah M (2002) Internal iliac aneurysm rupture into the rectum following endovascular exclusion: an unusual cause of massive lower gastrointestinal bleeding. $J$ Endovasc Ther 9: 907-911.

13. Lal N, Jairaj PS (1989) Iliac aneurysmo-colonic fistula. A case report and review of literature. Int Angiol 8: 151-153.

14. Saratzis N, Saratzis A, Melas N, Ktenidis K, Kiskinis D (2008) Aortoduodenal fistulas after endovascular stent-graft repair of abdominal aortic aneurysms: single-center experience and review of the literature. J Endovasc Ther 15: 441-448. 